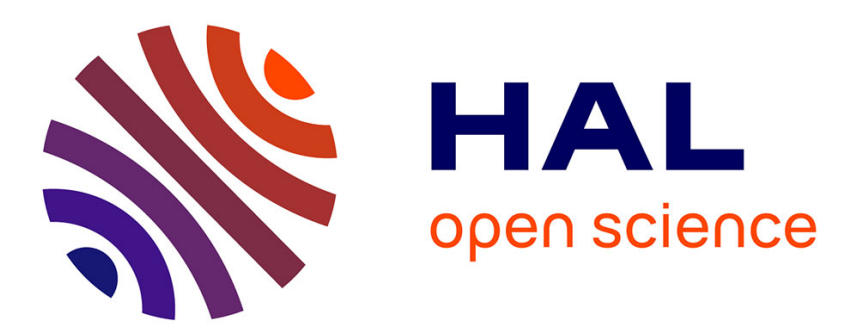

\title{
Étude typologique, technologique et culturelle de la céramique du Petit-Paulmy à Abilly (Indre-et-Loire)
}

\author{
Rémi Martineau
}

\section{To cite this version:}

Rémi Martineau. Étude typologique, technologique et culturelle de la céramique du Petit-Paulmy à Abilly (Indre-et-Loire). 29e colloque Interrégional sur le Néolithique, Feb 2009, Villeneuve d'Ascq, France. p. 479-494. halshs-00638312

\section{HAL Id: halshs-00638312 \\ https://shs.hal.science/halshs-00638312}

Submitted on 8 Mar 2012

HAL is a multi-disciplinary open access archive for the deposit and dissemination of scientific research documents, whether they are published or not. The documents may come from teaching and research institutions in France or abroad, or from public or private research centers.
L'archive ouverte pluridisciplinaire HAL, est destinée au dépôt et à la diffusion de documents scientifiques de niveau recherche, publiés ou non, émanant des établissements d'enseignement et de recherche français ou étrangers, des laboratoires publics ou privés. 


\title{
ÉTUDE TYPOLOGIQUE, TECHNOLOGIQUE ET CULTURELLE DE LA CÉRAMIQUE DU PETIT-PAULMY À ABILLY (INDRE-ET-LOIRE)
}

\author{
Rémi MARTINEAU
}

\section{INTRODUCTION}

La région du Grand-Pressigny a livré une telle quantité de vestiges lithiques que les rares découvertes de céramique sont presque passées inaperçues et que les corpus n'ont pas toujours été étudiés en détail. Par conséquent, nous ne connaissons que peu de choses sur l'organisation sociale, économique et culturelle des populations néolithiques qui ont occupé cette région. L'accent a davantage été mis sur les études lithiques, à l'échelle locale, nationale et internationale. Ces rares vestiges céramiques concernent neuf sites répartis dans les vallées de la Claise et de la Creuse (fig. 1). Ils ont déjà néanmoins fait l'objet d'une série de publications, concernant notamment des études typologiques (ARD \& Louboutin 2008, Berthouin 1980, CAuvin 1961, CoRdier 1961, GesLin et al. 1975, 1982a et b,

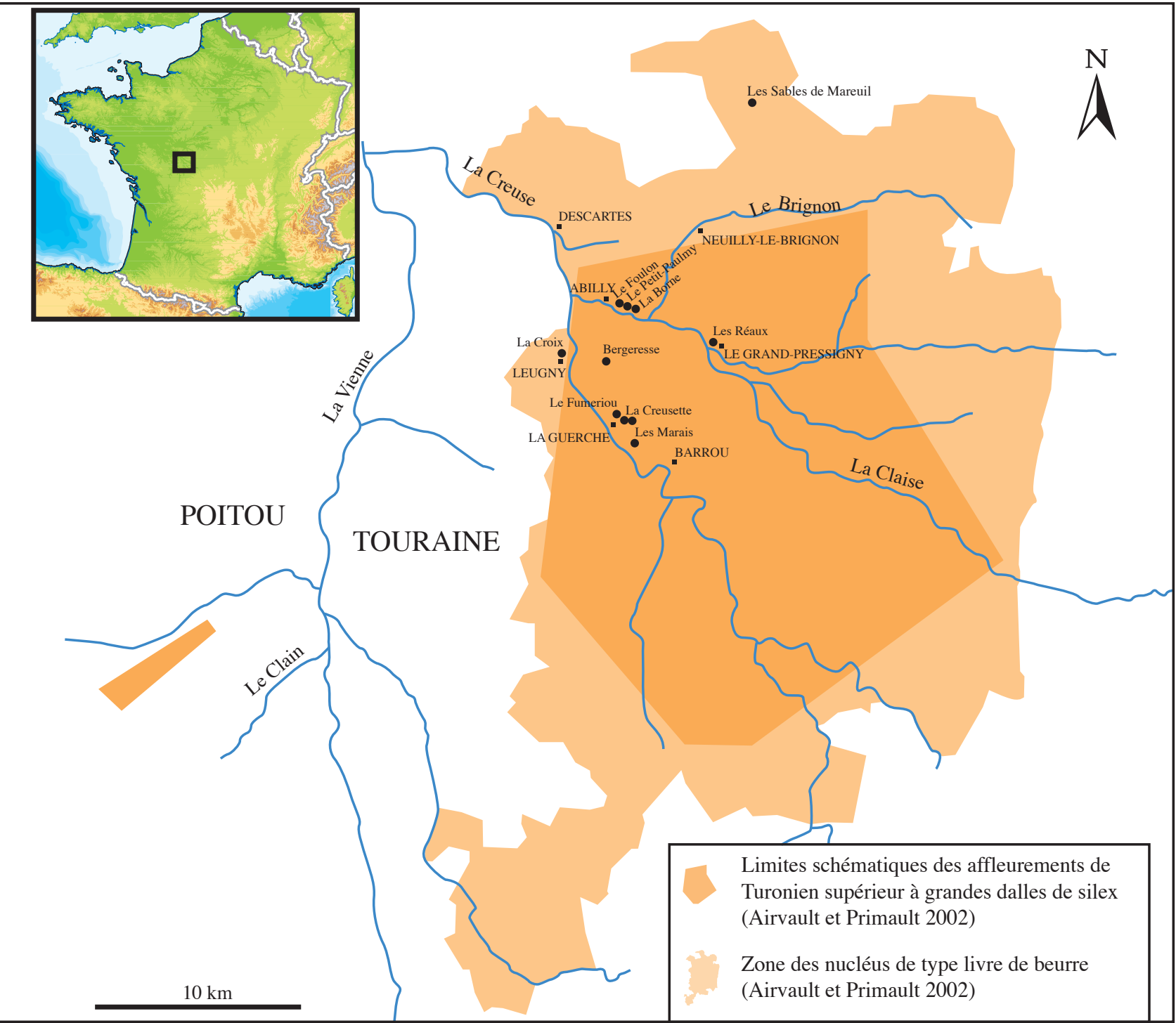

Fig. 1 - Carte de localisation des neuf sites de la région du Grand-Pressigny qui ont livré de la poterie du Néolithique final. Les sites de répartissent dans les vallées de la Claise et de la Creuse (DAO M-A. RODOT et R. MARTINEAU). 


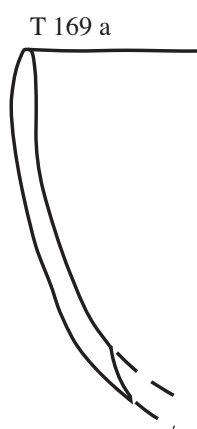

forme hémisphérique

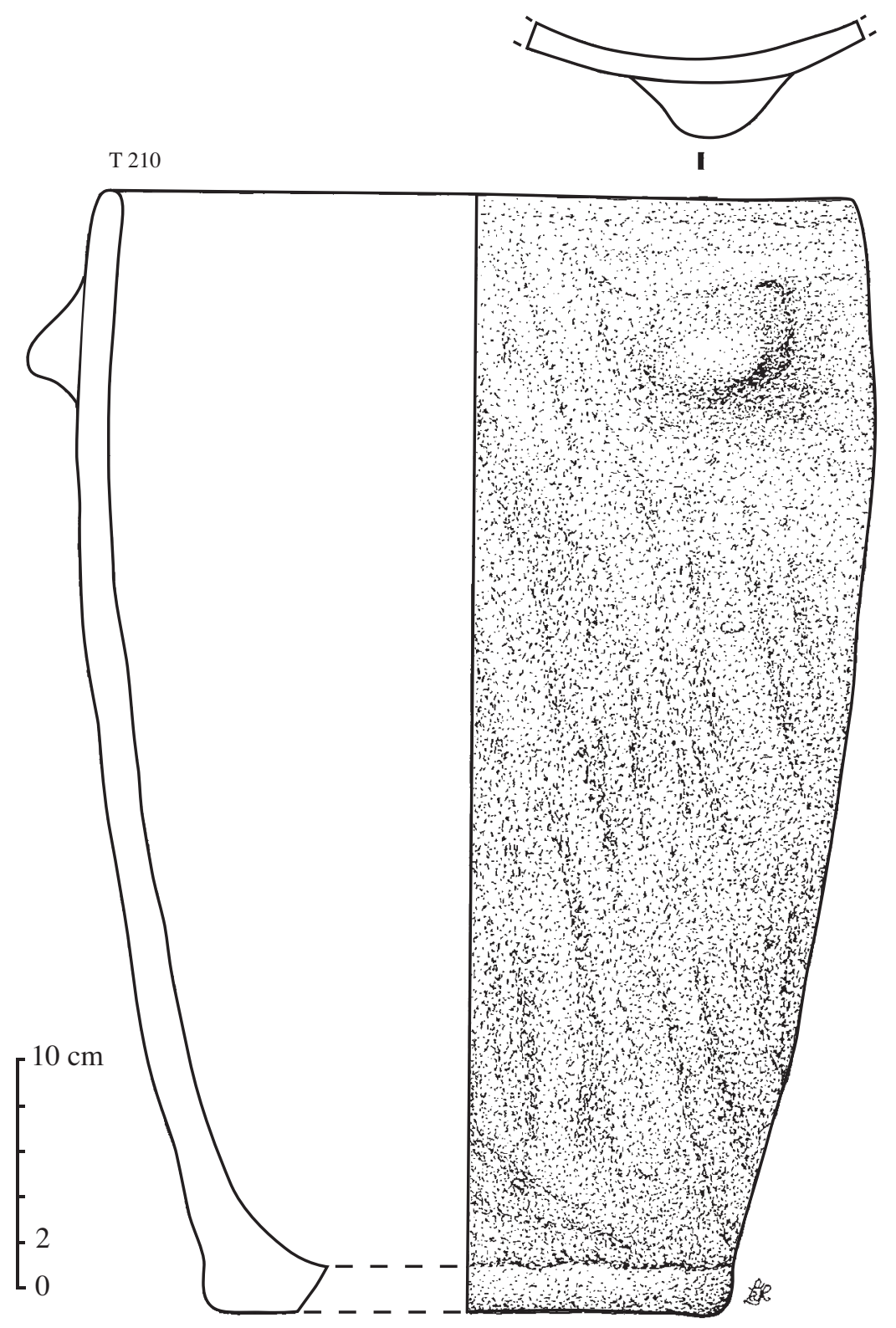

forme en tonneau à fond plat avec tenon de préhension

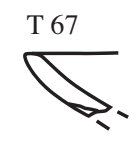

calotte de sphère

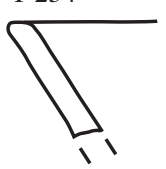

forme ouverte

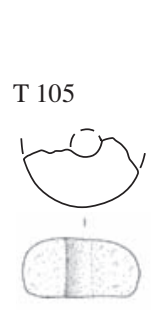

fusaïole

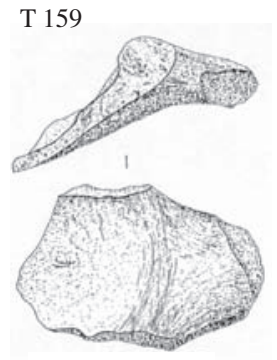

cuillère

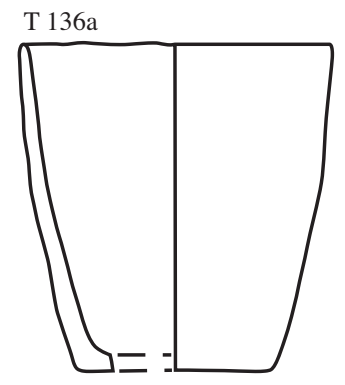

forme tronconique à fond plat

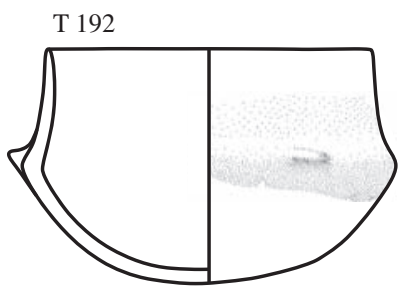

forme composite en $\mathrm{S}$, à fond rond, épaulement marqué et col concave, avec une languette de préhension

Fig. 2 - Synthèse typo-morphologique de la céramique du Néolithique final du Petit-Paulmy (dessins L-A. MILLET-RICHARD et R. MARTINEAU). 


\begin{tabular}{|c|c|c|c|c|c|c|c|c|c|c|c|c|}
\hline Sites & $\begin{array}{c}\text { Abilly Le } \\
\text { Petit- } \\
\text { Paulmy }\end{array}$ & $\begin{array}{c}\text { Barrou La } \\
\text { Creusette } \\
\text { diagnostic } \\
2000\end{array}$ & $\begin{array}{c}\text { La Guerche } \\
\text { Fumeriou }\end{array}$ & $\begin{array}{c}\text { Abilly Le } \\
\text { Foulon } \\
\text { fouilles } \\
\text { Millet- } \\
\text { Richard }\end{array}$ & $\begin{array}{l}\text { Abilly Le } \\
\text { Foulon } \\
\text { fouilles } \\
\text { Berthouin }\end{array}$ & $\begin{array}{c}\text { Barrou La } \\
\text { Creusette } \\
\text { atelier }\end{array}$ & $\begin{array}{c}\text { La } \\
\text { Guerche } \\
\text { La } \\
\text { Creusette }\end{array}$ & $\begin{array}{c}\text { Abilly } \\
\text { La } \\
\text { Borne }\end{array}$ & $\begin{array}{c}\text { Abilly } \\
\text { Bergeresse }\end{array}$ & $\begin{array}{c}\text { Leugny La } \\
\text { Croix }\end{array}$ & $\begin{array}{l}\text { Le Grand- } \\
\text { Pressigny } \\
\text { Les Réaux }\end{array}$ & $\begin{array}{c}\text { Barrou La } \\
\text { Creusette } \\
\text { dépôt de } \\
\text { lames }\end{array}$ \\
\hline $\begin{array}{c}\text { Sources } \\
\text { bibliogra- } \\
\text { phiques }\end{array}$ & $\begin{array}{l}\text { Martineau } \\
\text { à paraître }\end{array}$ & $\begin{array}{c}\text { Villes } \\
2001 \mathrm{a} \text { et } \mathrm{b} \text {, } \\
2003, \\
2006 \mathrm{~b}\end{array}$ & Villes 2002 & \begin{tabular}{|c|} 
Millet- \\
Richard \\
1995, \\
1997 \\
\end{tabular} & $\begin{array}{c}\text { Berthouin } \\
1980, \\
\text { Villes } 2003\end{array}$ & $\begin{array}{c}\text { Geslin et } \\
\text { al. } 1982 \mathrm{a} \\
\text { et } \mathrm{b}\end{array}$ & $\begin{array}{c}\text { Verjux } \\
1991\end{array}$ & $\begin{array}{c}\text { Cauvin } \\
1961\end{array}$ & $\begin{array}{c}\text { Verjux et al. } \text {. } \\
2009\end{array}$ & $\begin{array}{c}\text { Ard et } \\
\text { Louboutin } \\
2008\end{array}$ & $\begin{array}{c}\text { Cordier } \\
1961\end{array}$ & $\begin{array}{c}\text { Geslin } \text { et } \\
\text { al. } 1975\end{array}$ \\
\hline $\begin{array}{c}\text { Nombre } \\
\text { de tessons } \\
\text { dessinés } \\
\text { dans les } \\
\text { publica- } \\
\text { tions }\end{array}$ & 136 & 96 & - & 68 & 37 & 28 & 11 & 5 & 6 & 3 & 4 & 1 \\
\hline
\end{tabular}

Tab. I - Sites de la région du Grand-Pressigny comprenant de la céramique attribuée au Néolithique final.

Millet-Richard 1995, 1997 ; VerJux 1991, VerJux et al. 2009, VILLES 2001a et b, 2002, 2003, 2006b). Une première étude typo-technologique (MARTINEAU à paraître) et une étude pétrographique (CONVERTINI à paraître) ont déjà été réalisées sur le corpus du PetitPaulmy à Abilly. Un tableau permet de décompter le nombre d'individus provenant de chacun de ces contextes du Néolithiquefinal de la région du GrandPressigny (tab. I.). La rareté des vestiges céramiques est sans doute la raison pour laquelle plusieurs problématiques importantes n'ont pas encore pu être traitées, concernant notamment l'évolution chrono-culturelle et l'origine géographique des groupes qui ont occupé et exploité ce fameux silex du Grand-Pressigny. Avancer sur ces questions nécessite d'étudier exhaustivement et de manière très approfondie toutes les céramiques de tous les sites de cette région afin de les comparer, puis les intégrer à un contexte géographique plus large.

La céramique du Petit-Paulmy a été découverte en association avec un important amas de taille situé à mi-pente. La majeure partie du mobilier concerne l'industrie lithique, associée à une faible quantité de vestiges domestiques. La publication monographique donnera tous les détails concernant le site et son contexte (MARQUET \& MILLET-RICHARD 1995). Le matériel céramique est très fragmenté, très érodé et présente de nombreuses concrétions d'oxyde de manganèse. L'étude typologique et technologique approfondie a permis de quantifier les types de formes, de caractériser les techniques et de croiser quantitativement tous ces paramètres entre eux afin de constituer des groupes céramiques qui permettent de donner une image de la diversité typo-technique du corpus et de discuter de sa position chrono-culturelle régionale.

\section{TYPO-MORPHOLOGIE QUANTITATIVE ET CONSTITUTION DES GROUPES}

Les fragments de forme ont été rattachés à des types morphologiques par comparaison avec les types les plus complets. Le traitement quantitatif a été réalisé en tenant compte de deux calculs du nombre d'individus : le nombre minimum et le nombre maximum ; le nombre réel se situant sans doute entre ces deux estimations. Le nombre minimum (NMI) est calculé en évitant systématiquement les risques de doublons (RICE 1987, p. 292). Par exemple un fond plat et un moyen de préhension ou un bord qui pourraient appartenir au même type morphologique ne sont comptés qu'une fois. Tandis que le nombre maximum d'individus (NMAI) prend en compte tous ces individus pour le calcul, ne sachant pas s'ils appartiennent ou non à la même poterie. Le NMAI correspond en fait au nombre d'individus typologiques. Le NMAI du Petit-Paulmy est de 110 individus et le NMI est égal à 79 .

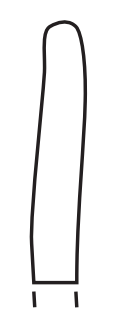

arrondie

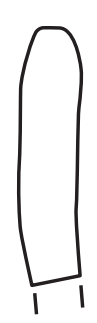

aplatie

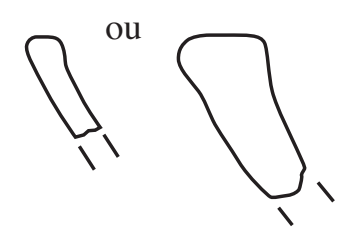

épaissie interne ou externe

$$
\mathbf{5 7 , 4} \% \quad 18,2 \% \quad 12,5 \%
$$

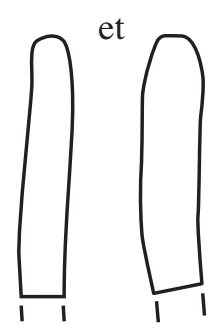

arrondie et aplatie

$3,4 \%$

Fig. 3 - Typologie des lèvres et répartition quantitative au sein du corpus (N=88). 
La synthèse des types morphologiques rencontrés sur le site fait état de huit types dont six concernent des poteries et deux des instruments en terre cuite (cuillère et fusaïole). Les poteries sont représentées par une forme en tonneau à fond plat pouvant comporter des moyens de préhension, une forme tronconique à fond plat (pl. 3, T136a), une forme composite en $S$ à fond rond avec col concave et languette de préhension (pl. 1, T192), une forme hémisphérique (pl. 4, T169a), une forme en calotte de sphère et une forme ouverte (fig. 2).

L'ensemble du mobilier étudié appartient au Néolithique final lato sensu. Mais à l'intérieur de cet ensemble, trois groupes typo-technologiques ont été constitués. Ces groupes de poteries (ou de tessons) sont appelés A, B et C.

Le groupe A comprend au minimum 55 individus et au maximum 86. Le groupe B (14 individus) concerne les formes en $\mathrm{S}$ avec un col concave et les formes ouvertes qui rappellent des types qui existaient déjà au Néolithique moyen. Le taux de brunissage et de battage de ces types est élevé et les épaisseurs sont caractéristiques comme nous le verrons plus loin. Le groupe $\mathrm{C}$ (10 individus) comprend des bords éversés et des lèvres aplaties épaissies caractéristiques. L'étude des conditions de cuisson permet d'appuyer l'attribution des poteries à l'un ou l'autre des groupes. L'obtention de tessons d'un gris moyen homogène résulte généralement de cuissons en atmosphère réductrice dans une structure fermée. Une grande partie de la céramique attribuée au groupe $C$ correspond à ces conditions de cuisson dont on sait qu'elles vont se développer très largement au cours de l'âge du Bronze. Ainsi, le groupe $C$ pourrait correspondre à la période de transition Néolithique final/âge du Bronze, le groupe $B$ au Néolithique final et le groupe A pourrait être soit contemporain du groupe B, soit très légèrement antérieur (début du Néolithique final ?).

Le corpus comprend 19 moyens de préhension, principalement représentés par des languettes horizontales. Une anse rostriforme permet de supposer un lien avec l'Artenac puisque ces anses sont caractéristiques de cette culture (pl. 2, T8). L'ensemble des moyens de préhension représente $17,3 \%$ du NMAI. Quatre types de lèvres ont été identifiés et se répartissent de la façon suivante : arrondies dans $57,4 \%$ des cas, aplaties pour $18,2 \%$, épaissies interne ou externe $12,5 \%$, arrondie et aplatie (sur la même poterie) dans 3,4 \% des cas (fig. 3).

Ces différents types ont été décomptés en fonction $\mathrm{du}$ NMI et du NMAI pour les trois groupes typotechnologiques auxquels le matériel a été attribué (MARTINEAU à paraître). Dans l'ordre décroissant et en pourcentage, le corpus des trois groupes réunis (NMAI) comprend des formes tronconique $(37,3 \%)$, hémisphérique $(30,9 \%)$, en S à col concave $(10,9 \%)$, en tonneau $(6,4 \%)$, en calotte de sphère $(1,8 \%)$, forme ouverte $(1,8 \%)$, puis les cuillères $(0,9 \%)$ et les fusaïoles $(0,9 \%)$ (fig. 4). Quelques différences de parts relatives apparaissent avec le traitement quantitatif du NMI. Ce ne sont alors plus les formes tronconiques mais les formes hémisphériques qui sont majoritaires. Cette différence s'explique par le fait que la forme tronconique est un peu surreprésentée lorsqu'on considère le nombre maximum d'individus car cette catégorie morphologique comprend à la fois des fonds, des bords et des moyens de préhension qui sont tous pris en compte dans ce mode de calcul. La différence entre les deux modes de calcul concerne également les parts relatives des calottes de sphère et des formes ouvertes qui sont très légèrement supérieures pour le NMI que pour le NMAI (fig. 4).

\section{LES PÂTES CÉRAMIQUES}

Basés sur la nature et l'angularité des inclusions, dixgroupesmacroscopiquesdepâteontétéidentifiés. Ils témoignent de la diversité des matériaux naturels qui ont été exploités. Aucun ajout d'inclusion n'a été observé. L'ensemble des groupes macroscopiques ne correspond sans doute qu'à une seule habitude de préparation des pâtes consistant à utiliser les matières premières locales sans les modifier. Les observations macroscopiques permettent en effet de supposer une origine locale pour ces matériaux qui comprennent principalement des quartz, des feldspaths, des muscovites et parfois de la biotite. L'absence de chamotte est à noter car elle est très fréquente dans l'Artenac.

\section{RECONSTITUTION DES CHAÎNES OPÉRATOIRES ET DES GROUPES TYPO-TECHNIQUES}

La répartition desépaisseursen fonction destypes morphologiques montre que les types tronconiques et en tonneau sont souvent plus épais $(11,6 \mathrm{~mm}$ en moyenne) que les formes hémisphériques $(7,6 \mathrm{~mm}$ en moyenne) ou en $S$ ( $8,1 \mathrm{~mm}$ en moyenne). Tandis que les formes à fond plat peuvent mesurer jusqu'à $22 \mathrm{~mm}$ d'épaisseur, les formes hémisphériques ou en $S$ ont des épaisseurs inférieures à 12 ou $14 \mathrm{~mm}$ selon les cas (fig. 5). Il existe donc bien une relation entre les formes et les épaisseurs des poteries.

Les chaînes opératoires ont été reconstituées grâce à l'identification des techniques de façonnage, de traitement de surface et de finition (MARTINEAU à paraître). Pour les quatre formes principales (tronconique, tonneau, hémisphérique et en $S$ ), douze chaînes opératoires ont pu être reconstituées (fig. 6). Pour les formes tronconiques $(\mathrm{N}=43)$ et en tonneau $(\mathrm{N}=7)$, il existe quatre chaînes opératoires 


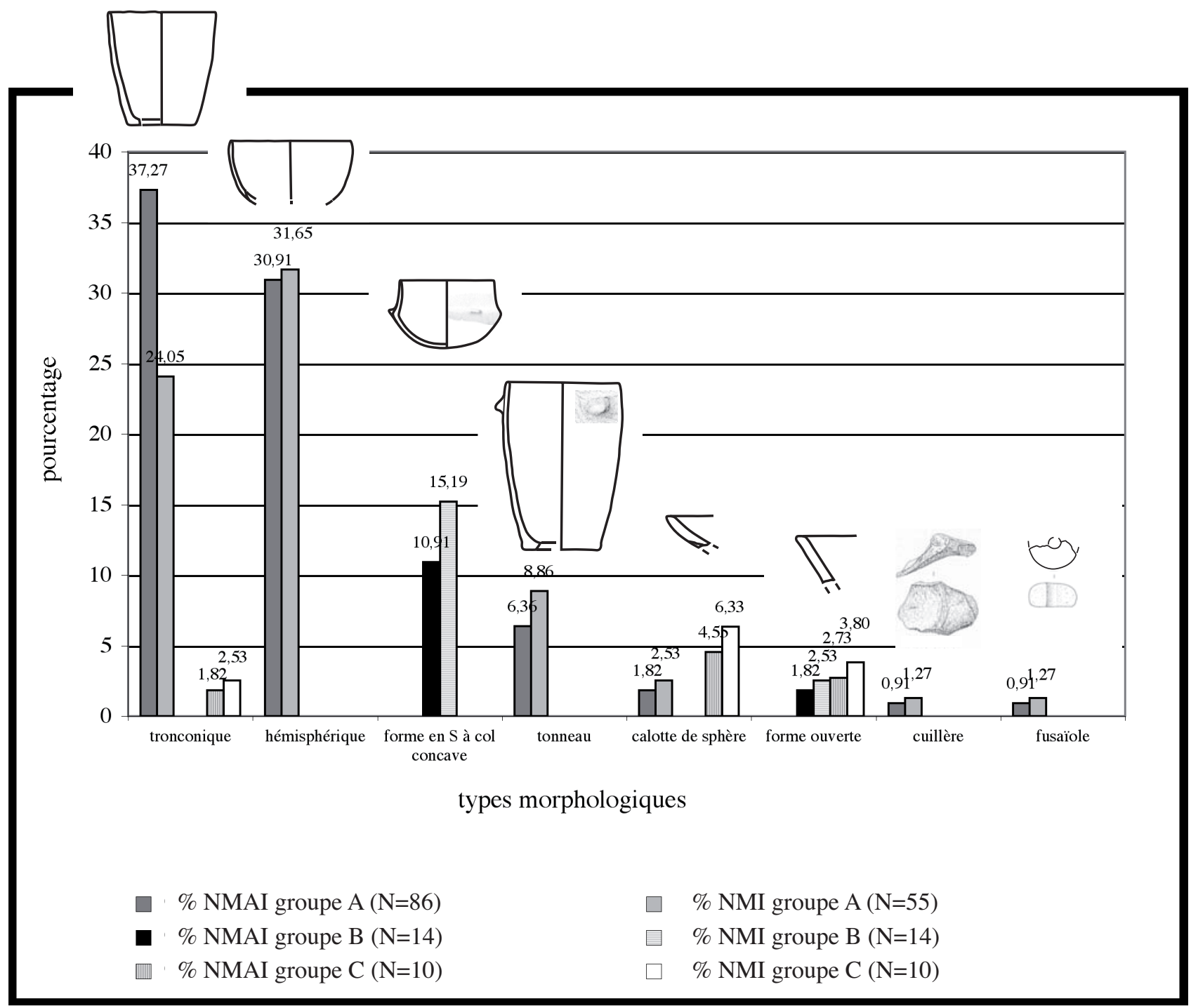

Fig. 4 - Répertoire morphologique quantifié des différentes phases chrono-culturelles. Le calcul a été réalisé à partir des attributions morphologiques. NMI : Nombre Minimum d'Individus (N=79). NMAI : Nombre Maximum d'Individus $(\mathrm{N}=110)$.

dont la plus simple correspond à des colombins peu modifiés au cours du montage et ne présente pas d'autres traitements de surface que le lissage. Elle concentre $86 \%$ des individus de ce type (fig. 6). Le raclage et le brunissage sont respectivement présents pour $6,5 \%$ de ces formes.

En ce qui concerne les formes hémisphériques $(\mathrm{N}=34)$, sept chaînes opératoires ont été identifiées, dont quatre principales. Les colombins ont été modifiés par des mises en forme donnant des structures étirées en S. 38,2 \% des poteries hémisphériques ne montrent aucun traitement de surfaceni aucunefinition (fig. 6). La chaîneopératoire 11 , comprenant battage et brunissage, concentre 20,5\% de ces poteries. La chaîne opératoire comprenant raclage, battage et brunissage représente 11,8\% des individus et la chaîne opératoire comprenant uniquement $\mathrm{du}$ brunissage comme finition représente aussi $11,8 \%$.
Les formes en $\mathrm{S}(\mathrm{N}=12)$ ont été fabriquées à partir de trois chaînes opératoires différentes, dont l'une comprend du brunissage uniquement et une autre $\mathrm{du}$ battage externe avec utilisation d'une contrebatte interne (MARTINEAU 2005) et du brunissage. Fabien Convertini a mis en évidence que les matériaux de cette poterie sont différents du reste du corpus et pourraient être exogènes (CONVERTINI à paraître, MARTINEAU \& CONVERTINI à paraître).

La chaîne opératoire 12 est identique à la 5 et la chaîne opératoire 13 identique à la 8 , ce qui fait qu'il n'y a pas quatorze mais douze chaînes opératoires différentes, dont deux sont partagées entre les formes en $S$ et hémisphériques. Ce fait renforce la proximité technologique entre ces deux formes dont les épaisseurs se répartissent également de façon assez semblable (fig. 5). Ces rapprochements sont confirmés par la présence de battage pour ces deux formes tandis qu'il est absent pour les formes 

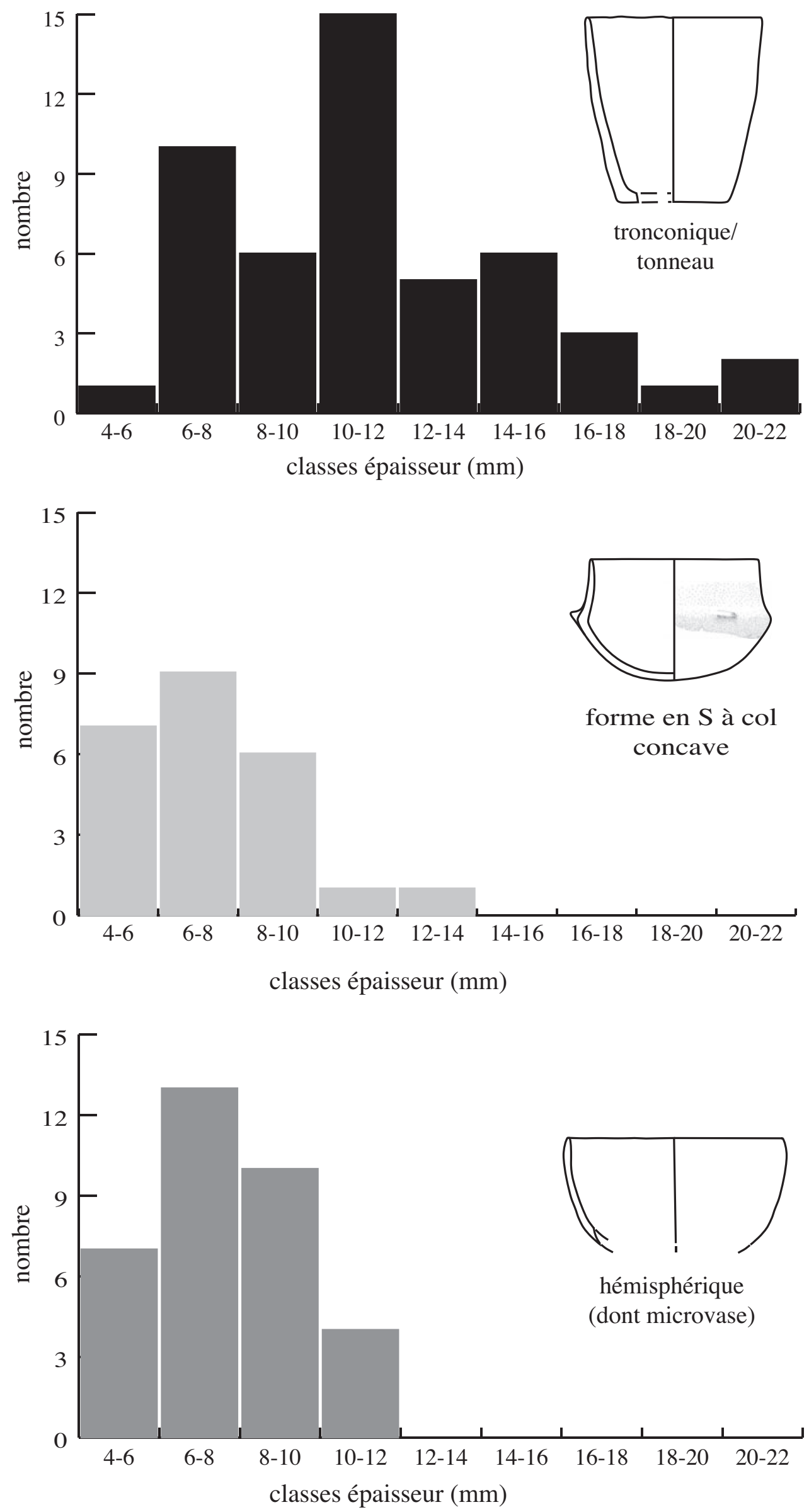

Fig. 5 - Répartition des classes d'épaisseur pour les trois principales formes céramiques du Petit-Paulmy. Il existe une bonne corrélation entre les formes et les épaisseurs (DAO R. MARTINEAU). 


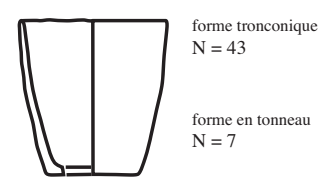

$\%$ / FORME

raclage $=6,5 \%$. pas de battage. brunissage $=6,5 \%$

ÉPAISSEUR MAXIMUM $(\mathrm{N}=135)$

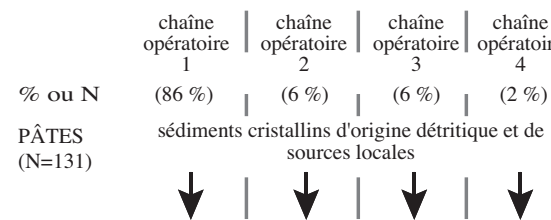

ÉBAUCHE
$(\mathrm{N}=82 / 110)$$\quad$ colombins peu modifiés

RACLAGE

$(\mathrm{N}=13 / 110)$

BATTAGE

EXTERNE

$(\mathrm{N}=16 / 110)$

BATTAGE

EXTERNE ET

INTERNE

$(\mathrm{N}=1 / 110)$

BRUNISSAGE

( $\mathrm{N}=28 / 110)$

CUISSON

$(\mathrm{N}=133)$
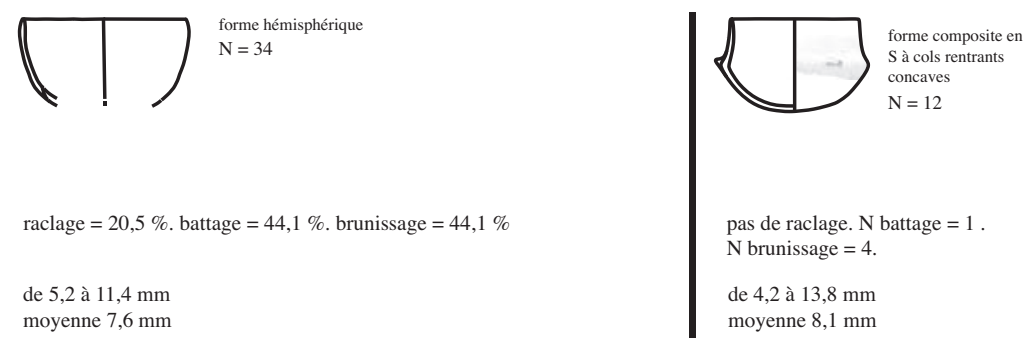

de 5,2 à $11,4 \mathrm{~mm}$
moyenne $7,6 \mathrm{~mm}$

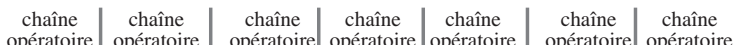

chaine |c chaîne $\mid \begin{gathered}\text { chaine } \\ \text { cón }\end{gathered}$

$\begin{array}{ccccccc}5 & 6 & 7 & 8 & 9 & 10 & 11 \\ (38,2 \%) & (5,9 \%) & (8,8 \%) & (11,8 \%) & (3 \%) & (11,8 \%) & (20,5 \%)\end{array}$

sédiments cristallins d'origine détritique et de sources locales
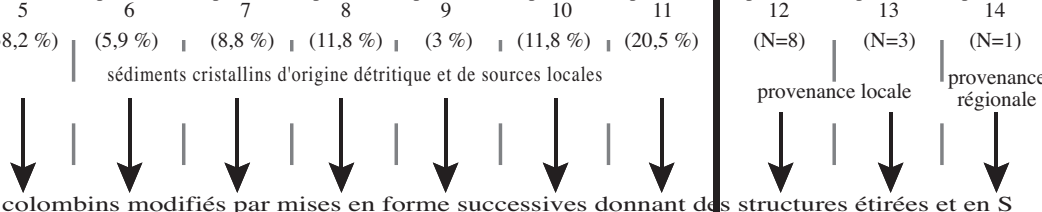

政

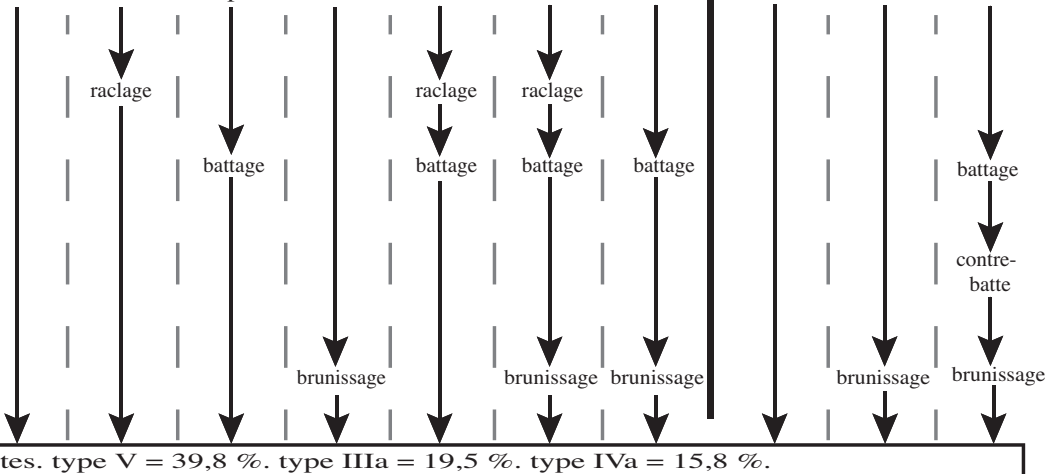

Fig. 6 -Synthèse des données technologiques et des chaînes opératoires concernant les trois principales formes (tronconique / tonneau, hémisphérique et en S). Douze chaînes opératoires différentes ont été reconstituées (DAO R. MARTINEAU).

à fond plat. Toutes ces observations permettent de proposer l'existence de deux ensembles techniques différents, l'un pour les formes tronconiques ou en tonneau à fond plat et l'autre pour les formes hémisphériques et en $\mathrm{S}$. Une partie des calottes de sphère pourrait sans doute être rattachée à ce second ensemble céramique.

\section{DISCUSSION}

\section{ENSEMBLES CÉRAMIQUES ET TRADITIONS TECHNIQUES}

Le groupe A rassemble deux ensembles techniques : les poteries à fonds ronds façonnées à partir de colombins étirés et comprenant une bonne fréquence des techniques de raclage, battage ou brunissage et les poteries à fonds plats à colombins peu étirés simplement lissées. Présents dans un même site sans stratigraphie ni structures, ces deux ensembles pourraient laisser supposer qu'il s'agit d'un mélange de deux périodes ou de deux cultures différentes. Il faut pourtant prendre en compte le fait que cette situation rassemblant ainsi ces deux ensembles techniques différents dans un même site est un cas de figure extrêmement fréquent au Néolithique final dans la moitié nord de la France. Le groupe du Gord par exemple rassemble systématiquement des poteries tronconiques à fonds plats dites " grossières », c'est-à-dire épaisses, et des poteries hémisphériques moins épaisses, dites « fines » (LAмвот 1981, Cottiaux 1989 et 1995). Ceci est d'ailleurs un phénomène général au Néolithique final en Europe occidentale (LAPORTE 1996).

On pourrait penser que la céramique du PetitPaulmy constitue un mélange de mobiliers du Néolithique récent (poteries à fonds plats) et du Néolithique final (poteries à fonds ronds). Mais l'absence d'armatures de flèches de type Sublaines (caractéristiques du Néolithique récent) au PetitPaulmy (communication orale Caroline Renard) constitue un argument majeur qui confirme notre hypothèse que l'ensemble de ce mobilier appartient au Néolithique final.

Il est néanmoins hautement probable que les céramiques à fonds plats soient culturellement héritées du Néolithique récent tandis que les poteries à fonds ronds correspondent à des apports nouveaux au Néolithique final et peut-être hérités d'autres sphères culturelles, notamment méridionales. De ce fait, à l'instar des «vents du nord et du sud» dans le Jura français (PÉTREQUIN 1993), les poteries tronconiques à fonds plats constitueraient un héritage du Néolithique récent, d'origine plutôt orientale, tandis que les poteries hémisphériques représenteraient un apport du Néolithique final d'origine méridionale. Ces influences culturelles méridionales ont d'ailleurs 
déjà été mises en évidence sur la façade atlantique (LAPORTE 2009). Ces deux grands courants culturels, oriental et méridional, semblent s'être rencontrés dans le Bassin parisien dans la première moitié du troisième millénaire avant notre ère.

Le site du Petit-Paulmy semble n'être qu'un des nombreux exemples de cette association de ces deux types d'ensembles céramiques dans un même site. En effet, une grande partie des sites du Néolithique final du Bassin parisien présente une telle association. Dans cette aire géographique, pendant la première moitié du troisième millénaire, on peut probablement parler de deux traditions céramiques distinctes, associées dans les mêmes contextes archéologiques. L'hypothèse que ces deux ensembles puissent correspondre à deux périodes chronologiques différentes est très difficilement soutenable à cause de la régularité de l'association de ces deux catégories de poteries. Si le cas n'était pas systématique, la situation nécessiterait d'être discutée car on pourrait alors envisager des mélanges de mobilier dus à l'absence d'une sédimentation suffisante pour mettreen placedescouchesdistinctes. Mais la récurrence de cette association dans tous les sites de cette période ne permet pas pour le moment de conclure que ces deux traditions représentent deux périodes chronologiques distinctes. Il s'agit davantage de deux traditions morphologiques et techniques différentes, héritées de deux courants culturels différents qui ont fusionné à un moment de l'histoire des sociétés néolithiques. Le processus de cette fusion, par acculturation ou par un autre processus, n'est pas connu et la fragilité des contextes archéologiques ne permet sans doute pas dans ce cas d'aller plus loin pour le moment.

Cette hypothèse de l'association de deux ensembles céramiques hérités de deux courants culturels différents ne contredit nullement l'idée que ces différences de formes, d'épaisseurs et de techniques puissent correspondre également à des différences fonctionnelles. Bien que cela reste entièrement à démontrer, il est probable que ces différences reflètent des savoir-faire différents liés à des habitudes culinaires différentes, ce qui relève tout autant de la culture que la forme des poteries. Ceci expliquerait d'ailleurs très bien l'excellente corrélation entre les types de forme et les épaisseurs des poteries qui jouent sans doute un rôle pour les poteries utilisées sur le feu. On pourrait imaginer que les poteries épaisses à fonds plats étaient davantage destinées à aller sur le feu que les formes hémisphériques à fonds ronds, moins épaisses.

Unetelle situation où deux ensembles céramiques très différents (et tout à fait comparables) sont associés dans un même contexte archéologique a déjà été observée dans d'autres régions au même moment. Nous pensons notamment au $\mathrm{XXXI}^{\mathrm{e}}$ siècle avant J.-C. dans le Jura où un cas tout à fait remarquable et comparable a été observé sur le lac de Chalain (Giligny 1994 et 1997, Giligny et al.
1995, PÉtrequin 1997). La différence dans ce cas est que les formes tronconiques à fond plat ont très rapidement disparu après avoir été pendant un temps très court associées aux formes à fond rond (station 3 de Chalain, couche IV). Toutes les formes de poterie, de toutes les dimensions, ont alors été fabriquées à partir d'une nouvelle tradition technique et culturelle, d'origine méridionale, produisant uniquement des fonds ronds (GILIGNY 1997, Martineau 2000). Malgré quelques décalages chronologiques entre les régions, ce phénomène semble avoir concerné une aire géographique considérable comprenant une grande partie du Bassin parisien. La région du Grand-Pressigny ne semble pas avoir fait exception à ce phénomène.

\section{ATTRIBUTIONS CHRONOLOGIQUES ET CULTURELLES}

Deux tessons permettent de supposer une très vraisemblable première occupation du site du Petit-Paulmy au Néolithique ancien, probablement au Villeneuve-Saint-Germain (VSG) (MARTINEAU à paraître). Sur le très proche site du Foulon, des datations radiocarbone permettent également de supposer une occupation au Néolithique ancien (Millet-Richard 1997). Il ne semble pas pourtant que du matériel en silex du Grand-Pressigny ait été mis en évidence sur des sites de cette période ( $\mathrm{N}$. Mallet, communication orale).

La majeure partie du mobilier du Petit-Paulmy est attribuable au Néolithique final et avec toute vraisemblance à la première moitié du 3e millénaire. Les éléments attribués au groupe B pourraient correspondre à une première phase d'occupation du site. Mais cet ensemble de céramiques peut être également tout à fait contemporain du matériel attribué au groupe $\mathrm{A}$, ce que les points communs techniques (raclage, battage, brunissage) entre ces deux groupes typo-techniques inciteraient à penser.

Plusieurs autres sites de la région du GrandPressigny sont comparables au Petit-Paulmy et attribuables au Néolithique final : "Le Foulon" (Berthouin 1980, Millet-Richard 1993, 1995 et 1997, VILles 2003) et "La Borne" (CAuvin 1961) à Abilly, "Les Réaux" au Grand-Pressigny (CORDIER 1961), "La Creusette" à Barrou (dépôt de lames, « habitat-atelier » et sondages VILLES 2000 ; GESLIN et al. 1975 et 1982a et b, VILLES 2001 et 2006b) et "La Croix" à Leugny (ARD \& LoubOutin 2008). Tous ces sites comprennent des poteries hémisphériques associées à des formes tronconiques et le plus souvent quelques cuillères et fusaïoles. Les études détaillées qui sont en cours sur ces sites confirment leur attribution au Néolithique final.

Les sites du Petit-Paulmy et du Foulon avaient été attribués au Vienne-Charente par comparaison avec des sites de la Vienne (Airvaux \& PrimaulT 2002). Par extension, le débitage des livres de beurre 
avait été également attribué à cette culture. Plus récemment, l'étude typologique et technologique de quatre sites du Vienne-Charente (ARD 2008) permet de mettre en évidence d'importantes différences avec la céramique du Petit-Paulmy ou du Foulon. L'absence de poteries hémisphériques et en $S$, brunies et/ou battues, constituent des différences notables avec la céramique du Petit-Paulmy et permettent d'exclure très certainement l'attribution de ce site au Vienne-Charente.

La question de l'attribution de la céramique des sites de la zone des affleurements de silex du Grand-Pressigny, au Gord ou à l'Artenac, a été débattue par L-A. MiLlet-Richard (1997) et A. VilLES (2003, 2006a et 2007). Des similitudes indéniables existent entre le Gord et la céramique de la région du Grand-Pressigny. Ce rapprochement culturel a été proposé depuis longtemps (GESLIN et al. 1982a). La région pressignienne doit-elle pour autant être entièrement rattachée au Gord ? Cette question se pose également pour le site du "Petit-Paulmy" pour lequel nous allons essayer de voir les différents arguments en faveur du rattachement à l'une ou l'autre culture.

L'anse rostriforme du Petit-Paulmy montre un lien culturel avec l'Artenac (pl. 2, T8) mais ne suffit pas à attribuer la totalité du site à cette culture. La forme en $S$ à col concave (pl. 1, T192) existe aussi dans l'Artenac. On peut considérer que le groupe B rassemble les éléments qui se rapprochent le plus de l'Artenac. Mais dans l'Artenac de "Diconche", les cuillères n'apparaissent qu'après 2600 cal. BC (Burnez \& Fouéré 1999). Au "Petit-Paulmy", l'absence d'assiettes à décor interne et de carènes sinueuses et la rareté des anses rostriformes inciteraient plutôt à comparer le corpus avec l'Artenac I des zones périphériques, défini par C. Burnez (Burnez et al. 1998, Burnez \& Fouéré 1999, p. 257). Néanmoins, la diversité typologique de l'Artenac ne se retrouve pas dans l'assemblage céramique du Petit-Paulmy et les éléments diagnostics caractéristiques de l'Artenac y restent très rares.

Il faut reconnaître que les caractéristiques de ce corpus céramique sont en revanche assez comparables à celles du groupe du Gord qui comprennent de fréquentes formes tronconiques à fonds plats, hémisphériques ou en calottes de sphère, de rares profils discontinus à cols rentrants et toujours quelques cuillères et fusaïoles (LAMBOT 1981, Cottiaux 1989 et 1995). L'ensemble du groupe $B$ et une partie du groupe A s'insèrent assez bien dans ce groupe culturel. Les sites du groupe du Gord restent malheureusement trop rares et souvent assez mal conservés, ce qui limite la connaissance que nous avons de cette culture.

Les pâtes céramiques du site éponyme du Gord ne contiennent de la chamotte que de façon tout à fait anecdotique (CotTIAux 1989 et 1995). Notons que cette détermination n'a pas fait l'objet d'analyses microscopiques et que la présence de ces inclusions reste à vérifier. Ceci milite plutôt en faveur d'un rapprochemententrelePetit-Paulmy, dontles poteries ne contiennent pas de chamotte, et le groupe du Gord. Mais quelle est la part réellement représentée par la chamotte dans les différents sites du groupe du Gord ? La fréquence importante de chamotte dans les poteries de "La Bouchardière" à Monts (RODOT 2007 et MARTINEAU inédit), situé à $45 \mathrm{~km}$ au nord-ouest du Grand-Pressigny, et surtout sa très grande fréquence dans l'Artenac (CONVERTINI 1996 et 1999, RODOT 2007) constituent des différences techniques qui renforcent les rapprochements culturels entre le groupe du Gord et le Petit-Paulmy. Néanmoins, il reste encore difficile de trancher sur la question du rattachement du site du Petit-Paulmy à l'une ou l'autre de ces deux cultures (Gord ou Artenac). Peut-être même faut-il envisager que ce site ait pu appartenir à une autre culture archéologique?

\section{CONCLUSION ET PERSPECTIVES}

Cette première étude approfondie d'un corpus céramique dans la région du Grand-Pressigny donne une vision qualitative et quantitative de la typo-morphologie des céramiques du PetitPaulmy. Deux ensembles céramiques bien distincts appartenant à un même groupe technique (A) ont été mis en évidence. La diversité des chaînes opératoires est à la fois le reflet de la faible standardisation de ces productions, notamment des formes tronconiques épaisses, et d'une variabilité vraisemblablement liée à la production domestique et peut-être individuelle des poteries dans le site. La faible standardisation des productions céramiques est une caractéristique de tous les sites du Néolithique final de la moitié nord de la France, excepté pour les gobelets campaniformes. Au-delà de cette diversité, c'est surtout la mise en évidence de quelques caractères technologiques communs entre ces deux ensembles techno-morphologiques qui permet, avec la récurrence de leur association et l'absence d'armatures de type Sublaines dans les sites d'habitat, de supposer et de proposer leur contemporanéité. Cette situation constitue un problème important et préalable à l'étude de la plupart des problématiques concernant la céramique et la chronologie du Néolithique final de la région Centre et du Bassin parisien. Et ce ne sont certainement pas les corpus céramiques des sépultures collectives qui permettront de résoudre ces questions. L'étude très détaillée et exhaustive de chaque corpus de la région du Grand-Pressigny s'impose comme une nécessité afin de permettre des comparaisons précises et des attributions culturelles discutées sur des bases solides. 

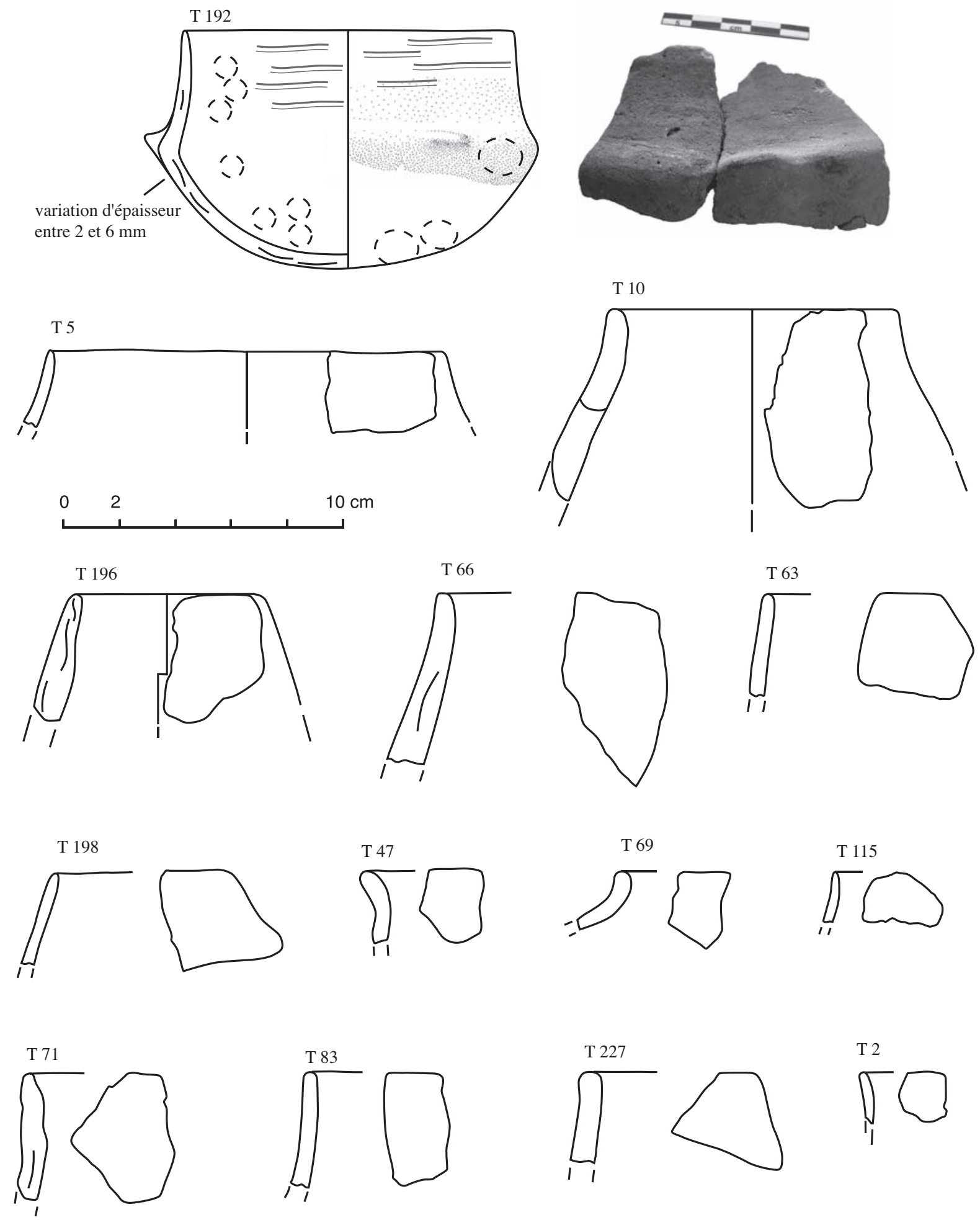

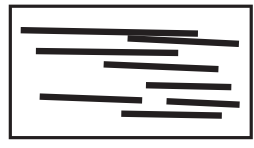

grattage/raclage à consistance plastique

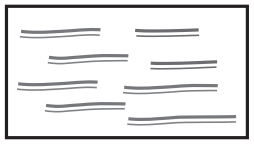

brunissage à consistance plastique dur ou cuir

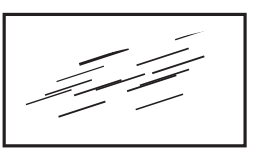

lissage à la main mouillée

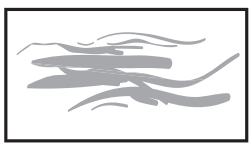

empreintes de végétaux
, méplat externe

1-)

dépressions circulaires internes

P1. 1 - Poterie du "Petit-Paulmy" (Abilly, Indre-et-Loire) attribuée au Néolithique final. Forme en S à col concave et languette sur épaulement. Bords rentrants à profil concave pouvant se rattacher à ce type de forme (dessins R. MARTINEAU et C. Touzel). 
T 136a
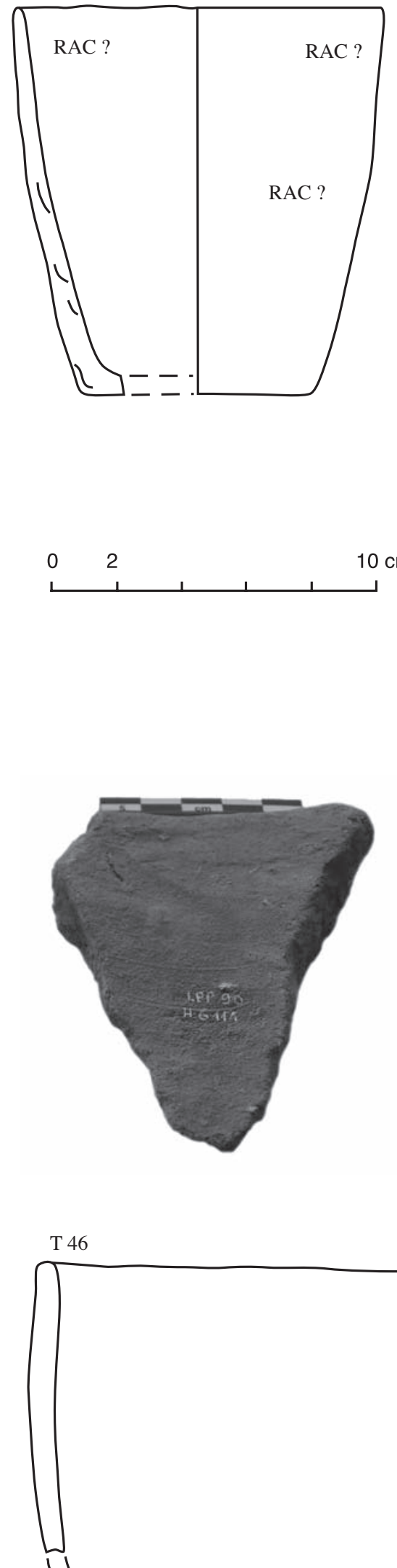

T 136b
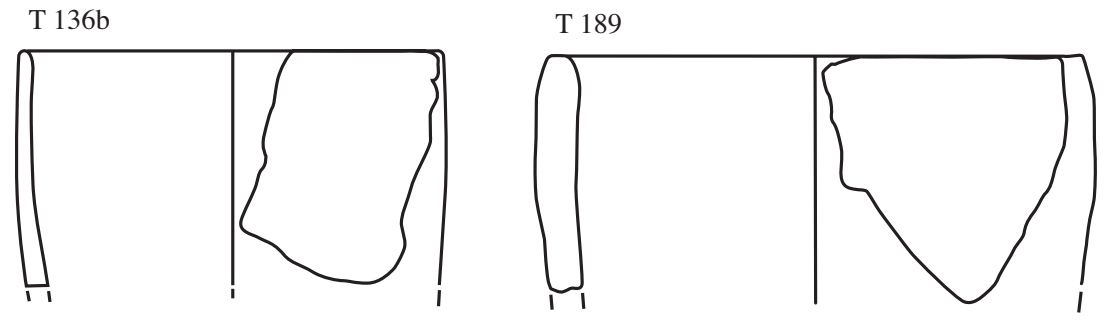

T 80
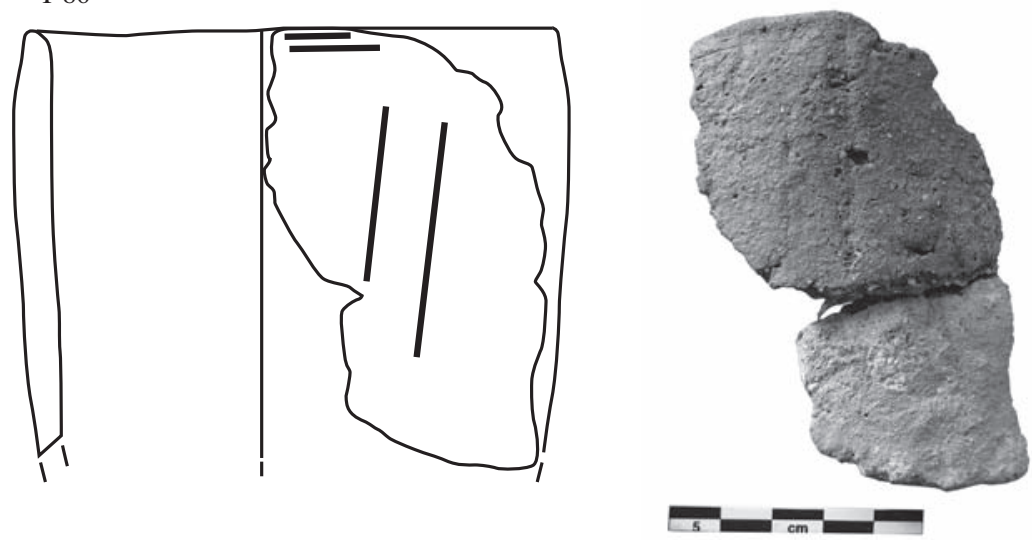

$\mathrm{T} 1$
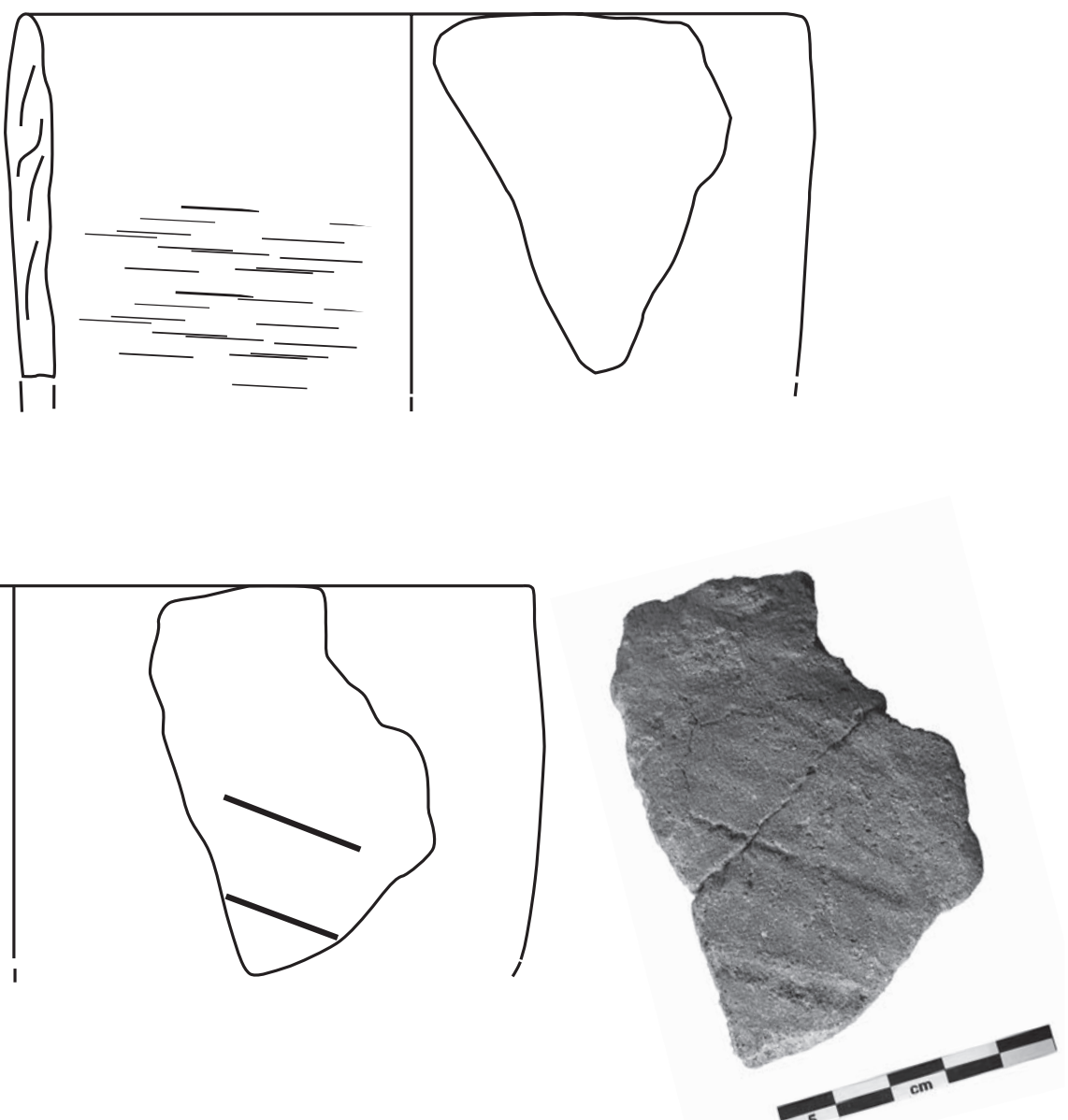

P1. 2 - Poterie du "Petit-Paulmy" (Abilly, Indre-et-Loire) attribuée au Néolithique final. Formes en tonneau et moyens de préhension (dessins R. MARTINEAU). 

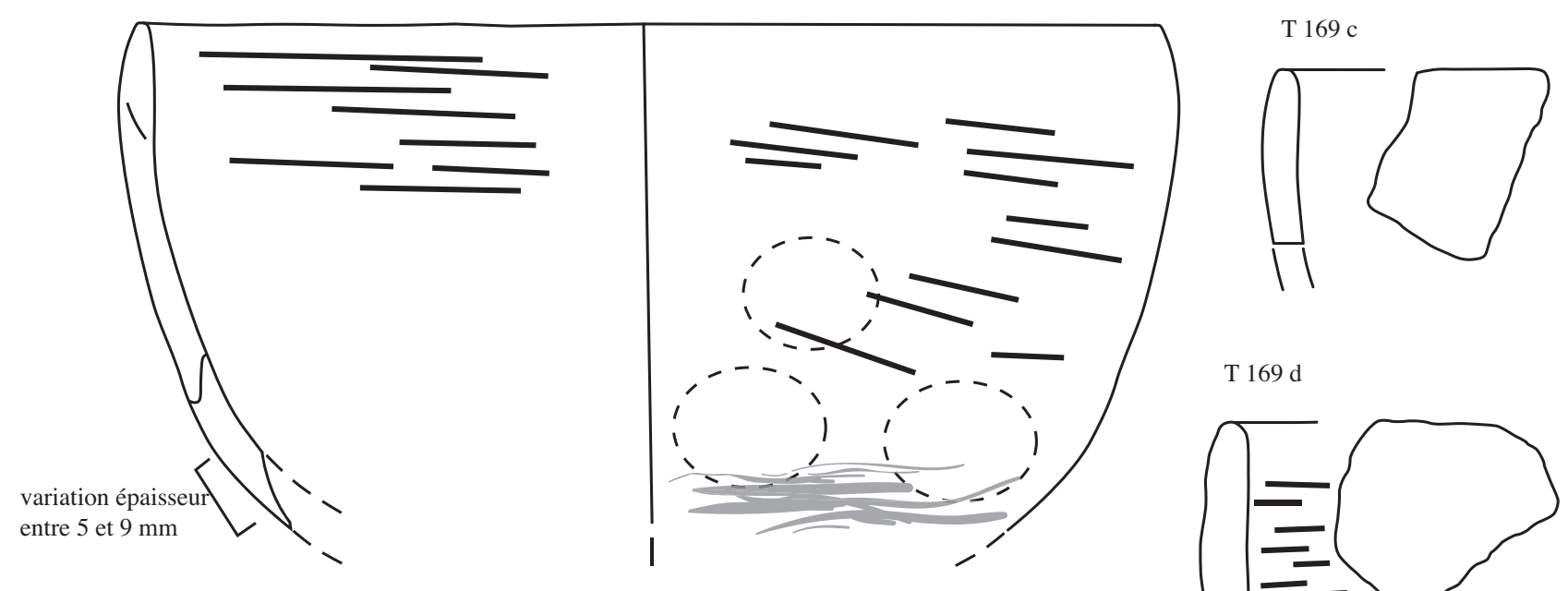

T $169 \mathrm{~d}$

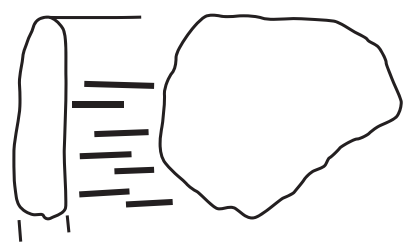

$\mathrm{T} 169 \mathrm{~b}$
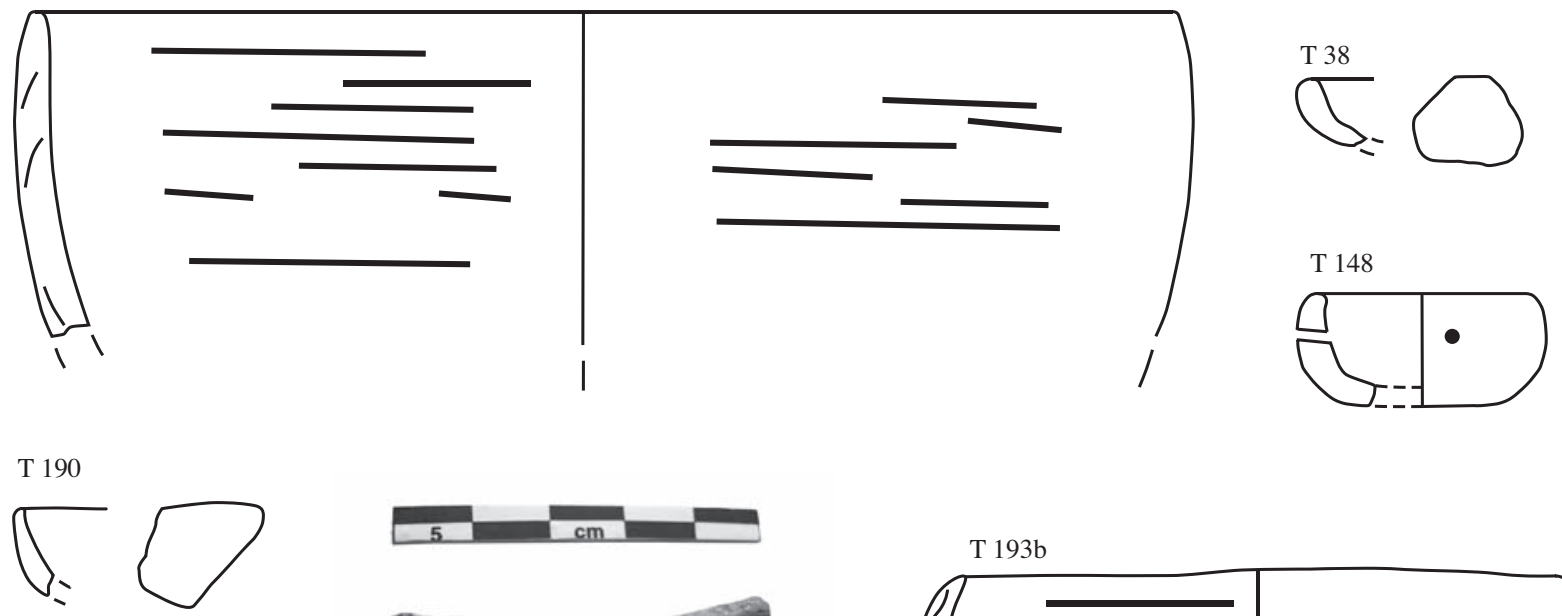

T 232
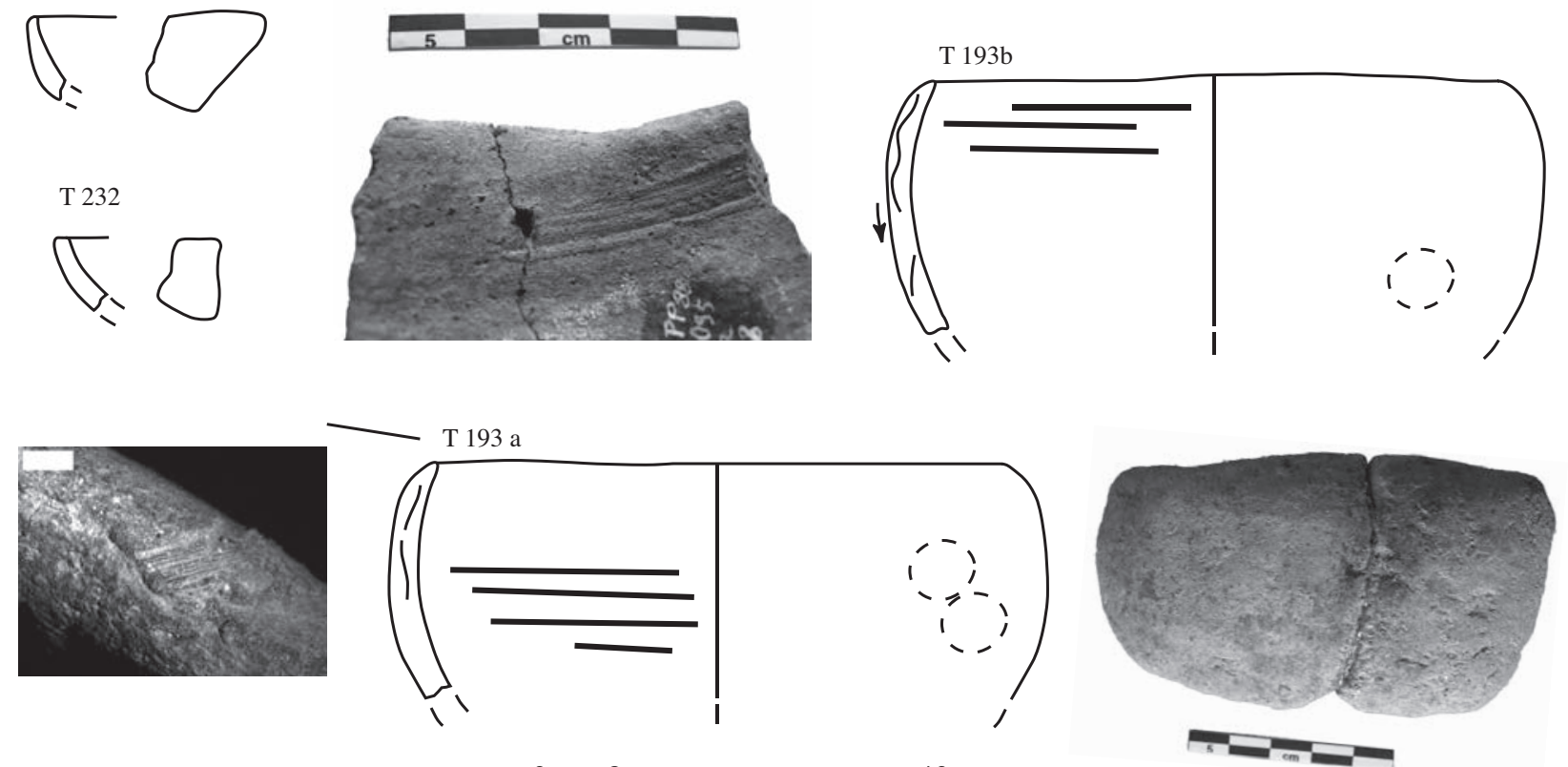

Pl. 3 - Poterie du "Petit-Paulmy" (Abilly, Indre-et-Loire) attribuée au Néolithique final. Formes tronconiques ou cylindriques (dessins R. MARTINEAU). 

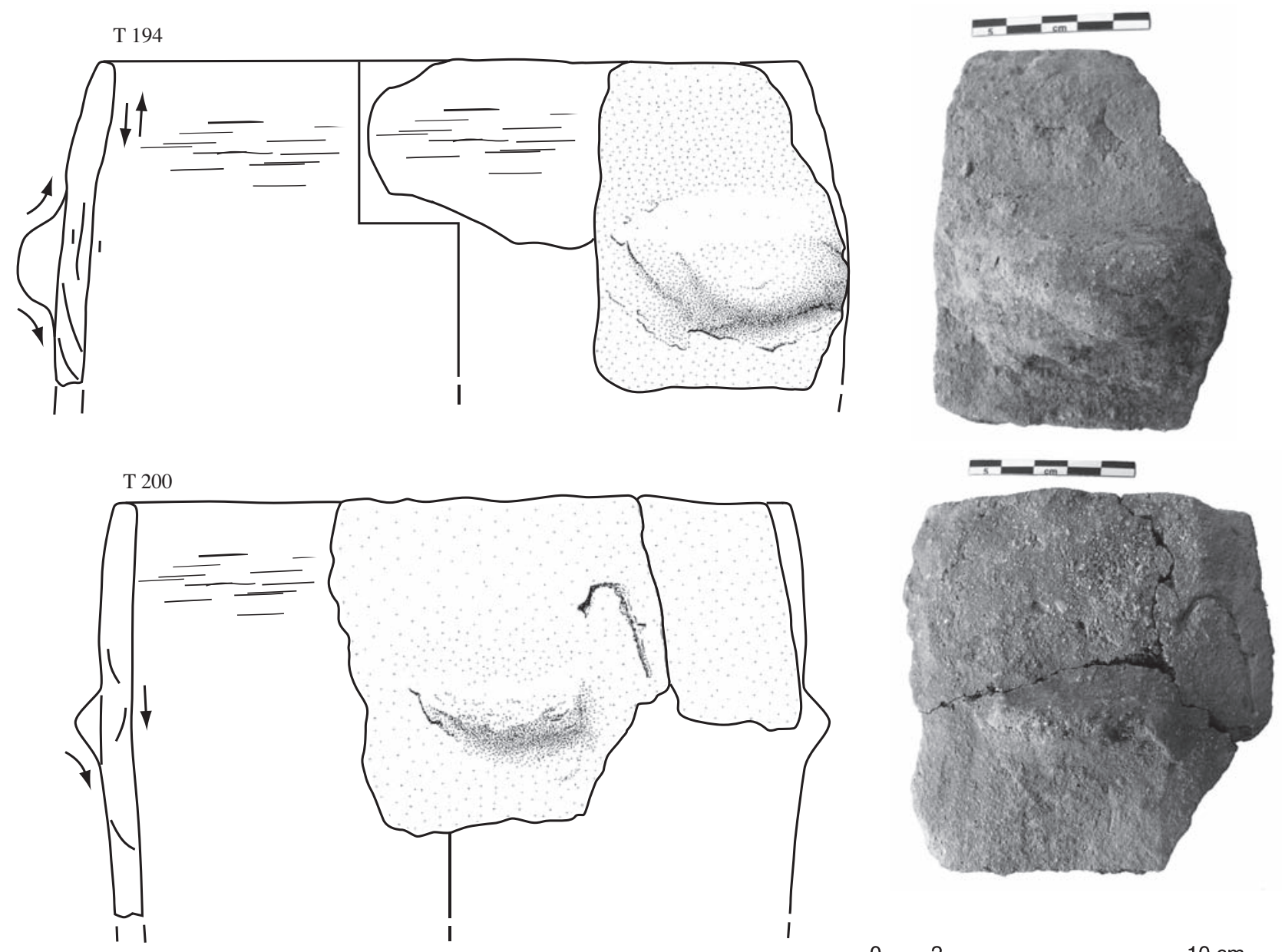

$\mathrm{T} 131$
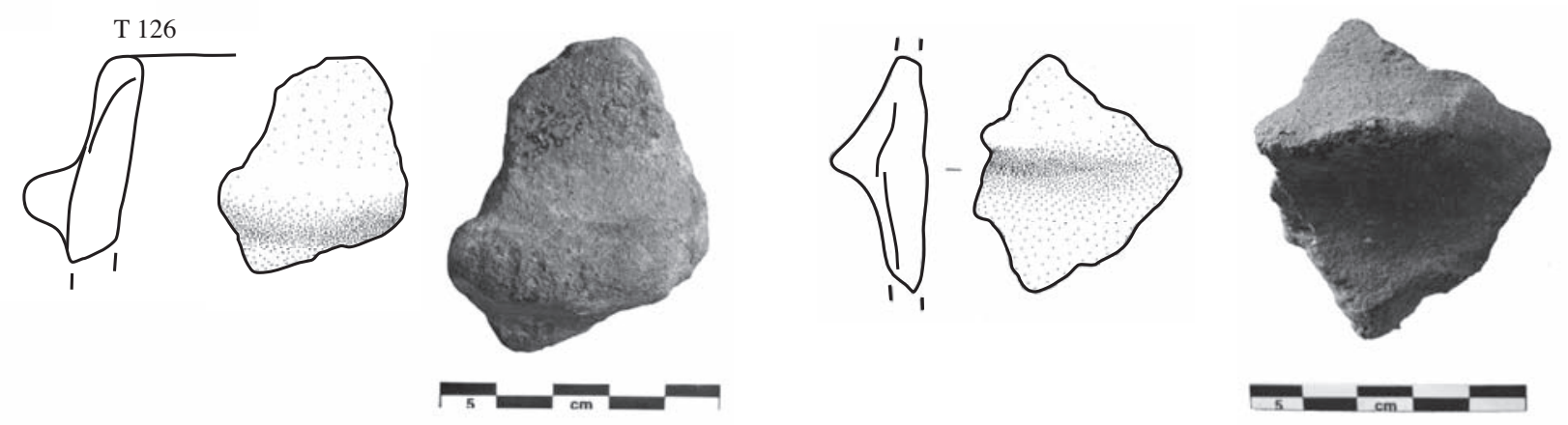

$\mathrm{T} 133$
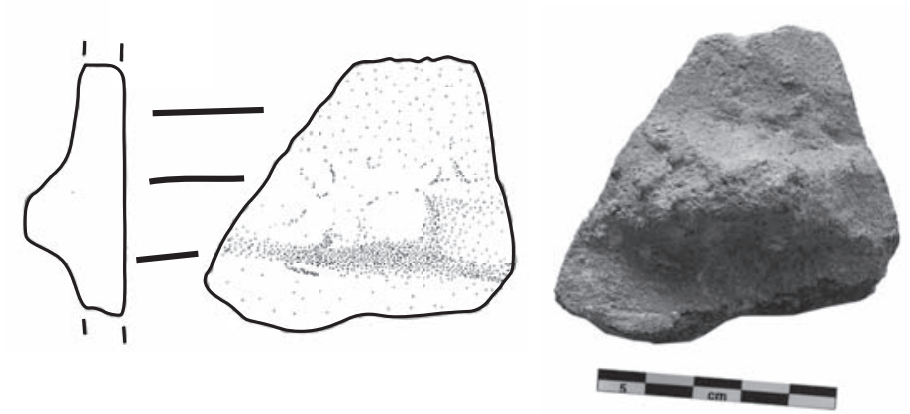

T 8

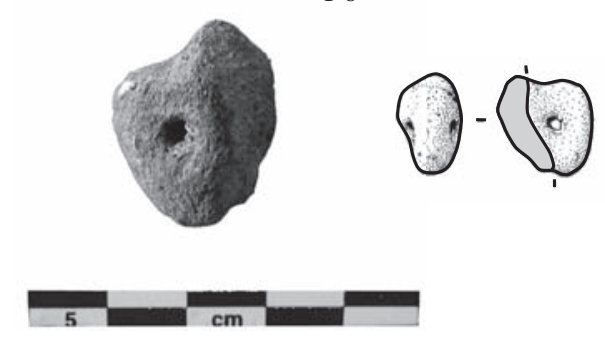

Pl. 4 - Poterie du "Petit-Paulmy" (Abilly, Indre-et-Loire) attribuée au Néolithique final. Formes hémisphériques. Les traces de raclage et de battage sont fréquentes (dessins $R$. MARTINEAU et C. TOUZEL). 


\section{Remerciements}

Jean-Claude Marquet nous a confié l'étude de la céramique du "Petit-Paulmy" et a financé une partie des analyses. Laure-Anne Millet-Richard a réalisé deux des dessins publiés dans cette étude. Une partie de ces travaux a été développée dans le cadre du Programme Collectif de Recherche Le Néolithique final au Grand-Pressigny, coordonné par Alain Villes et financé par le Service Régional de l'Archéologie de la Région Centre. Ces recherches ont été soutenues par la Fondation Fyssen.

\section{BIBLIOGRAPHIE}

ARD Vincent (2008) - «Traditions techniques et savoirfaire céramiques au Néolithique récent dans le Centreouest de la France : le cas des sites d'habitat attribués au Vienne-Charente ", Bulletin de la Société préhistorique française, 105, 2, p. 345-369.

ARD Vincent, LOUBOUTIN Catherine, avec la contribution de BOURGUEIL B. \& MILLET-RICHARD L-A. (2008) - « Leugny "La Croix" (Vienne) : une enceinte néolithique des bords de Creuse ", Bulletin des Amis du Musée de Préhistoire du Grand-Pressigny, 59, p. 15-29.

AIRVAUX Jean \& PRIMAULT Jérôme (2002) " Considérations sur l'extension géographique du Néolithique final à " livre de beurre » en Touraine et Poitou (France) », L'Anthropologie, 106, p. 269-294.

BERTHOUIN Fernand (1980) - " Habitat néolithique du Foulon, Abilly, Indre-et-Loire », Bulletin de la Société préhistorique du Grand-Pressigny, 31, p. 14-17.

BURNEZ Claude \& FOUÉRÉ Pierrick (dir.). (1999) - Les enceintes néolithiques de Diconche à Saintes (CharenteMaritime). Une périodisation de l'Artenac. Société préhistorique française, mémoire $n^{\circ} \mathrm{XXV}$ et Association des Publications chauvinoises, mémoire $n^{\circ} \mathrm{XV}$, vol 1 et 2 .

BURNEZ Claude, FOUÉRÉ Pierrick \& LOUBOUTIN Catherine (1998) - " Artenac et Campaniforme dans le Centre-ouest de la France ", Bulletin de la Société préhistorique française, 95, 3, p. 303-304.

CAUVIN Marie-Claire (1961) - « Découverte de céramique au Grand-Pressigny », L'Anthropologie, 65, p. 181-184.

CONVERTINI Fabien (1996) - «Étude pétrographique et techno-culturelle de la céramique néolithique » dans BURNEZ Claude (dir.) - Le site des Loups à Échiré (DeuxSèvres), ed. Musée des Tumulus de Bougon et Conseil Général des Deux-Sèvres, p. 225-232.

CONVERTINI Fabien (1999) - « Analyse pétrographique et technologique de la céramique » dans BURNEZ Claude \& FOUÉRE Pierrick (dir.) - Les enceintes néolithiques de Diconche à Saintes (Charente-Maritime). Une périodisation de l'Artenac. Société préhistorique française, mémoire $\mathrm{n}^{\circ} \mathrm{XXV}$ et Association des Publications chauvinoises, mémoire $\mathrm{n}^{\circ} \mathrm{XV}$, vol 1 et 2, p. 195-207.

CONVERTINIFabien (à paraître) - «Étude pétrographique de la céramique du Petit-Paulmy » dans MARQUET JeanClaude \& MILLET-RICHARD Laure-Anne (dir.) - Le site Néolithique final du Petit-Paulmy à Abilly (Indre-et-Loire), Supplément à la Revue Archéologique du Centre.
CORDIER Gérard (1961) - « Le fond de cabane néolithique des Réaux au Grand-Pressigny (Indre-et-Loire) », Gallia Préhistoire, 4, p. 183-192.

COTTIAUX Richard (1989) - Étude de la céramique du site éponyme de Compiègne "Le Gord" (Oise), mémoire de maîtrise de l’Université de Paris I, non publié.

COTTIAUX Richard (1995) - « La céramique du site éponyme du "Gord" à Compiègne (Oise) », Bulletin de la Société préhistorique française, 92, 1, p. 97-106.

GESLIN Michel, BASTIEN Gilbert \& MALLET Nicole (1975) - « Le dépôt de grandes lames de La Creusette, Barrou, (Indre-et-Loire) », Gallia Préhistoire, 18, 2, p. 402422.

GESLIN Michel, BASTIEN Gilbert, MALLET Nicole \& FRESLIER Bernard (1982a) - " Le site de La Creusette, habitat et atelier ", Bulletin de la Société préhistorique du Grand-Pressigny, 33, p. 24-33.

GESLIN Michel, BASTIEN Gilbert, MALLET Nicole, FRESLIER Bernard \& GENTY Pierre (1982b) - « Le petit atelier de taille de La Creusette (commune de Barrou, Indre-et-Loire) », Bulletin de la Société préhistorique française, 79, 10-12, p. 399- 410.

GILIGNY François (1994) - «Variabilité et transferts techniques dans le Jura à la fin du $\mathrm{IV}^{\mathrm{e}}$ et au III ${ }^{\mathrm{e}}$ millénaires avant J.-C. " dans Terre cuite et société. La céramique, document technique, économique, culturel, XIVe Rencontres Internationales d'Archéologie et d'Histoire d'Antibes, Juan-les-Pins, APDCA, p. 363-380.

GILIGNY François, 1997 - « La céramique de Chalain 3 » dans PÉTREQUIN Pierre (éd.) - Les sites littoraux néolithiques de Clairvaux-les-Lacs et de Chalain (Jura). III, Chalain station 3, 3200-2900 avant J.-C., Paris : Maison des Sciences de l'Homme, vol. 2, p. 327-370.

GILIGNY François, MARECHAL Denis, PETREQUIN Pierre, PETREQUIN Anne-Marie \& SAINTOT Sylvie (1995) - « La séquence néolithique final des lacs de Clairvaux et de Chalain (Jura). Essai sur l'évolution culturelle » dans VORUZ Jean-Louis (éd.) - Chronologies néolithiques. De 6000 à 2000 avant notre ère dans le Bassin rhodanien. Actes du colloque d'Ambérieu-enBugey, 19-20 sept. 1992, Ambérieu-en-Bugey : Société Préhistorique Rhodanienne, (Documents du Département d'Anthropologie de l'Université de Genève ; 20), p. 313-346.

LAMBOT Bernard (1981) - « Le site chalcolithique du Gord à Compiègne (Oise) - Note préliminaire ", Cahiers archéologiques de Picardie, 8, p. 5-18.

LAPORTE Luc (1996) - «Quelques réflexions sur le Néolithique final du Centre-ouest de la France », Revue archéologique de l'Ouest, 13, p. 51-74.

LAPORTE Luc (2009) - « Discussions et synthèse. Le Néolithique récent et final de la façade maritime des Charentes dans son contexte local, régional et national » dans LAPORTE Luc (dir.) - Des premiers paysans aux premiers métallurgistes sur la façade atlantique de la France (3500-2000 avant J.-C.), Mémoire XXXIII, Association des Publications Chauvinoises, p. 683-753.

MARQUET Jean-Claude \& MILLET-RICHARD LaureAnne (1995) - «L'habitation-atelier Néolithique final du "Petit-Paulmy" à Abilly (Indre-et-Loire). Présentation préliminaire », Revue archéologique de l'Ouest, supplément $\mathrm{n}^{\circ} 7$, p. $247-271$. 
MARTINEAU Rémi (2000) - Poterie, techniques et sociétés. Études analytiques et expérimentales à Chalain et à Clairvaux (Jura), entre 3200 et 2900 avant J.-C., thèse de doctorat, UFR des Sciences de l'Homme, du Langage et de la Société, Archéologie et Préhistoire, Université de Franche-Comté, Besançon, non publié.

MARTINEAU Rémi (2005) - « Identification of the "beater and anvil" technique in Neolithic context : experimental approach » dans LIVINGSTONE-SMITH Alexandre, BOSQUET Dominique \&MARTINEAU Rémi(ed.)-Pottery manufacturing process : reconstruction and interpretation, XIVth Congress of the UISPP, Liège, Belgium, 2-8 sept. 2001, British Archaeological Reports (BAR), International Series 1349, p. 147-156.

MARTINEAU Rémi (à paraître) - " Typologie et technologie de la céramique du "Petit-Paulmy" (Abilly, Indre-et-Loire) » dans MARQUET Jean-Claude \& MILLERICHARD Laure-Anne (dir.) - Le site Néolithique final $d u$ "Petit-Paulmy" à Abilly (Indre-et-Loire), Supplément à la Revue Archéologique du Centre.

MARTINEAU Rémi \& CONVERTINI Fabien (à paraître) - « Croisement des données typologiques et pétrographiques » dans MARQUET Jean-Claude \& MILLET-RICHARD Laure-Anne (dir.) - Le site Néolithique final du "Petit-Paulmy" à Abilly (Indre-et-Loire), Supplément à la Revue Archéologique du Centre.

MILLET-RICHARD Laure-Anne (1993) - «Rapport de la fouille programmée sur le site du Foulon à Abilly ", Bulletin des amis du Musée de Préhistoire du Grand-Pressigny, 44, p. 34-42.

MILLET-RICHARD Laure-Anne (1995) - « L'habitat néolithique final du Foulon à Abilly (Indre-et-Loire) » dans Actes du colloque interrégional sur le Néolithique, Évreux 1993, Revue archéologique de l'Ouest, supplément $\mathrm{n}^{\circ} 7$, p. 233-245.

MILLET-RICHARD Laure-Anne (1997) - Habitats et ateliers de taille au Néolithique final dans la région du Grand-Pressigny (Indre-et-Loire). Technologie lithique, thèse de doctorat de l'Université de Paris I Panthéon Sorbonne, non publié.

PÉTREQUIN Pierre (1993) - « North wind, south wind. Neolithic technical choices in the Jura Mountains, 37002400 BC » dans LEMONNIER Pierre - Technological Choices. Transformation in material cultures since the Neolithic, Routledge, Londres et New-York, p. 36-76.

PÉTREQUIN Pierre (dir.) (1997) - Les sites littoraux néolithiques de Clairvaux-les-Lacs et de Chalain (Jura), III, Chalain station 3, 3200-2900 avant J.-C., Paris, Maison des Sciences de l'Homme, 2 vol., 765 p.

RICE Prudence M. (1987) - Pottery analysis. A sourcebook, The University of Chicago, Chicago et Londres.
RODOT Marie-Angélique (2007) - Les matériaux céramiques au Néolithique final dans le Centre et le Centre-Ouest de la France: natures, provenances et habitudes techniques, thèse de doctorat de l'Université de Bourgogne, non publié.

VERJUX Christian (1991) - «Fouille de sauvetage sur un atelier de taille du silex du Néolithique final dans la région du Grand-Pressigny (Indre-et-Loire) » dans actes du XVecolloque interrégional sur le Néolithique, Châlons-surMarne, 22-23 oct. 1988, Voipreux, p. 173-181.

VERJUX Christian, WEISSER Stella, RODOT MarieAngélique \& LEROY Damien (2009) - «Découverte de vestiges domestiques en contexte d'ateliers de taille du silex du Néolithique final à Abilly "Bergeresse" (Indre-etLoire) », Bulletin des amis du Musée de Préhistoire du GrandPressigny, 60, p. 17-44.

VILLES Alain (2001a) - Résultats de la fouille d'évaluation du site de "La Creusette" à Barrou (Indre-et-Loire), Rapport de fouilles, Service régional de l'Archéologie de la Région Centre, non publié.

VILLES Alain (2001b) - « Résutat de la fouille d'évaluation du site de "La Creusette" à Barrou (Indre-et-Loire). Première partie : la céramique », Buletin des Amis du musée de Prégistoire du Grand-Pressigny,52, p. 47-57.

VILLES Alain (2002) - La Guerche - "Fumeriou" (Indreet-Loire), Rapport de sondages, Service régional de l'Archéologie de la Région Centre, non publié.

VILLES Alain (2003) - «Que savons-nous des affinités culturelles du Grand-Pressigny au Néolithique récent et final ? ", Bulletin des amis du Musée de Préhistoire du GrandPressigny, 54, p. 43-74.

VILLES Alain (2006a) - " La question de l'attribution chrono-culturelle des sites du Néolithique récent et final de la région du Grand-Pressigny (Indre-et-Loire) » dans BARAY Luc (dir.) - Artisanats, sociétés et civilisations : hommage à Jean-Paul Thevenot, $24^{\mathrm{e}}$ supplément à la Revue archéologique de l'Est, S.A.E., p. 217-249.

VILLES Alain (2006b) - « Évaluations archéologiques sur le complexe néolithique du Grand-Pressigny : le site de Barrou "La Creusette" (Indre-et-Loire). Résultats préliminaires du diagnostic réalisé en 2000 »dans DUHAMEL Pascal (dir.) - Impacts interculturels au Néolithique moyen. Du terroir au territoire: sociétés et espaces, $25^{\mathrm{e}}$ supplément à la Revue archéologique de l'Est, S.A.E., p. 353-382.

VILLES Alain (2007) - «Quel est, aujourd'hui, le « vrai visage » du Grand-Pressigny ? » dans ÉVIN Jacques (dir.) - Un siècle de construction du discours scientifique en Préhistoire, Vol. 3 «... Aux conceptions d'aujourd'hui ",

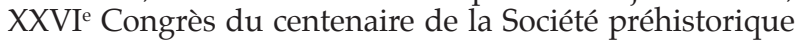
française, Avignon, 21-25 sept. 2004, p. 311-330.

\title{
L'auteur
}

\author{
Rémi MARTINEAU \\ CNRS, UMR 5594, ArTeHis Archéologie, Terre, Environnement, Histoire \\ Université de Bourgogne \\ UFR Sciences de la Terre, \\ 6 bd Gabriel \\ F - 21000 Dijon \\ remi.martineau@u-bourgogne.
}




\title{
Résumé
}

Une étude qualitative et quantitative a permis d'évaluer le nombre minimum et le nombre maximum d'individus céramiques attribués au Néolithique final. Le corpus comprend trois groupes typo-techniques qui correspondent à trois phases chrono-culturelles.

Une quantification des différents types morphologiques permet de donner une image du répertoire céramique du site au Néolithique final. Les matériaux utilisés pour la fabrication des poteries sont d'origine locale. Il existe une bonne corrélation entre les épaisseurs et les formes des poteries, mais aussi avec les différentes techniques (lissage, raclage, battage, brunissage) utilisées pour leur production. Les poteries des trois formes principales ont été fabriquées à partir de douze chaînes opératoires différentes qui ont été reconstituées en détail. Le corpus est majoritairement représenté par deux grands ensembles typo-techniques qui semblent appartenir à deux grandes traditions céramiques présentes dans une grande partie de la moitié nord de la France. La contemporanéité de ces deux traditions, ainsi que l'attribution culturelle du site, sont discutées.

Mots-clefs : Néolithique final - Grand-Pressigny - Poterie - Technologie céramique - Chaîne opératoire

\begin{abstract}
For the Late Neolithic period, the Minimum Number of Individuals (MNI) and the Maximum Number of Individuals (MaNI) have been estimated by a qualitative and a quantitative study. Three typo-technical groups have been highlighted, probably a showing chrono-cultural distinction.

A quantitative study of pottery morphology gives a good picture of the ceramic assemblage of the site. Raw materials used for the pottery production come from a local area. Twelve chaînes opératoires have been reconstructed in detail. A good correlation between pottery thickness, morphological types and technical characteristics confirms the validity of the groups constituted. The two ceramic assemblages of the site correspond to two different traditions which are present in a large area of Northern France, coming respectively from the east and the south. The very probable contemporaneity of these two assemblages in a lot of Late Neolithic sites in this area helps us to interpret these data. Taking into account all the technical, typometric and stylistic parameters, the attribution to a Neolithic culture is also discussed.
\end{abstract}

Keywords : Late Neolithic - Grand-Pressigny - Pottery - Ceramic technology - Chaîne opératoire

\section{Zusammenfassung}

Anhand einer qualitativen und quantitativen Studie wurden die minimale und die maximale Anzahl der dem Endneolithikum zugeordneten Keramikindividuen ermittelt. Der Corpus umfasst drei typologische und technische Gruppen, die drei chronologischen und kulturellen Phasen entsprechen.

Eine Quantifizierung der unterschiedlichen Formen vermittelt ein Bild des endneolithischen Keramikrepertoires des Fundplatzes. Der für die Herstellung der Keramik benutzte Ton ist lokaler Herkunft. Es existiert eine Korrelation zwischen der Dicke der Wandung, der Form der Gefäße und den unterschiedlichen Herstellungstechniken (Glättung, Schaben, Schlagen, Kieselglättung). Die Gefäße der drei Hauptformen wurden in zwölf unterschiedlichen, im Detail rekonstruierten Arbeitsschritten hergestellt. Der Corpus besteht überwiegend aus zwei umfangreichen, durch typologische und technische Kriterien definierte Ensembles. Diese scheinen zwei bedeutenden in Nordfrankreich weitverbreiteten Keramik-Traditionen anzugehören. Neben der Frage, ob diese beiden Traditionen zeitgenössisch sind, wird die kulturelle Zuweisung des Platzes diskutiert.

Schlagwörter : Endneolithikum - Grand-Pressigny - Töpferei - Keramiktechnologie - Arbeitsschritte.

Traduction : Isa ODENHARDT-DONVEZ (donvezservit@wanadoo.fr). 